\title{
Mystik und Bantu-Frömmigkeit
}

\author{
Von HARALD VON SICARD
}

\section{Prolegomena}

Ein Afrikanist wird unvermutet vor das Problem „Mystik in Bantu-Afrika“ gestellt. Er ist überrascht und verwirrt. In den mehr als vierzig Jahren meiner Beschäftigung mit Afrika bin ich weder in der Mission, noch in der Religionsforschung, noch in der Ethnologie auf das Wort „Mystik“ gestossen und habe auch niemals etwas von Bantu-Mystik gehört. Ein Bantu-Wort dafür gibt es nicht, was aber natürlich nicht auszuschliessen braucht, dass das Ding selbst bei den Bantu vorhanden ist.

Nur drei Arbeiten sind mir bekannt, deren Titel ausdrücklich von afrikanischer Mystik spricht: I) Der 2. Band von J. Cremers Les Bobo (Paris 1927 ) hat den. Untertitel La mentalité mystique, 2) I963 veröffentlichte der Afrikaner Mukoko-Mokeba in der in Yaounde (Kamerun) erscheinenden Zeitschrift Abbia, revue culturelle camerounaise (Heft 3-4, S. 39 ff.) den Aufsatz „Philosophical Basis of Bakwiri Mysticism“ und 3) das „Colloque du Centre de Recherches d'Histoire des Religions de l'Université de Strasbourg“" (Paris 1965) behandelte Réincarnation et vie mystique en Afrique Noire. Wer hier aber etwas von Mystik zu finden erwartet, wird schwer enttäuscht.

Eine gewisse Begriffsverwirrung liegt hier vor, die mit Placide Tempels' Philosophie bantoue (1949) einen ihrer Höhepunkte erreichte. Afrikaner und Nichtafrikaner wetteifern darum, abstrakte Begriffe, die ursprünglich von Europäern aus europäischem Verständnis heraus für europäische Erscheinungen und Bedürfnisse geprägt wurden, im allgemeinen recht unkritisch auf afrikanisches Leben und afrikanische Erscheinungen zu übertragen, ohne danach zu fragen, wieweit dadurch dem Tatbestand Zwang angetan wird oder nicht. Wir haben es hier mit jenen unbewussten "Lügen“ von Subjektivismen zu tun, wie der Ethnologe Vajda sie genannt hat (Traditionelle Konzeption und Realität in der Ethnologie, Festschr. Ad. E. Jensen I964, Bd. II, S. 774). Der Beobachter ,,ist nicht im Stande, sich von den. Vor- 
stellungen der Kulturwelt frei zu machen, in und von der er erzogen wurde", oder - um es anders auszudrücken - mit einem Problem, das an die allgegenwärtige Semantik grenzt. Wir müssen aber auch gleich wieder hinzufügen, dass sich diese Problemstellung bei uns entwickelte, als sich die Diskussion von dem jetzt überwundenen Subjekt-Objekt-Denken und seiner Problematik, die doch beide in sehr hohem Masse gerade auch in die Mystik hineingreifen, der Polarität von Sprache und Welt zuwandte.

In der Feststellung der ,unbewussten Lüge" braucht nicht unbedingt nur eine Abwertung zu liegen, ist doch dieses Einzwängen letzten Endes ein Übersetzungsversuch derer, die das zu Úbersetzende im besten Falle nur notdürftig kennen. Und doch leidet die ganze Arbeit unter diesem Versuch, denn man sieht die Dinge nicht so, wie die Afrikaner selbst es tun und wie sie ihnen wichtig sind.

Es verhält sich ja nicht so, als wollten wir den Afrikanern etwas so „Feines“ und „Tiefes“ wie die Mystik nicht zugestehen, als steckte hier also noch ein Rest kolonialen Denkens und seiner von den Afrikanern belächelten kindischen europäischen Selbstüberschätzung, so wie man es etwa noch in der 3. Aufl. der RGG (Bd. IV, r960) nachlesen kann: „Die Mystik hat ihre Wurzeln in der primitiven Religion" (F. Heiler), beileibe nicht mehr! Viele von uns stehen heute solchen veralteten Entwicklungsvorstellungen fremd gegenüber, und wer auch nur ,,dem Entwicklungsdenken nicht a priori den Rang eines für sämtliche Aspekte menschlicher Existenz gültigen Dogmas zuerkennt, wird eine verhältnismässig hochstehende Form der Religion in der Frühzeit der Menschheit nicht von vornherein ausschliessen " (K. Narr, Urgeschichte der Kultur, 196r, 7). Das aber gilt in noch viel höherem Grade von der Mystik in der Bantu-Frömmigkeit.

\section{Die Bantu-Frömmigkeit}

Als Ausgangspunkt unserer Betrachtung wähle ich Richters Behauptung in der RGG3 (IV, Sp. I238): „Erst wenn die unmittelbare Subjekt-Objekt-Einheit des mythischen Stadiums der Religion verloren wurde und nach der klassischen Zeit der ersten religiösen Offenbarung, die den Menschen im Kosmos isoliert und den Abgrund zwischen Gott und Mensch aufreisst, tritt die Mystik auf", um den Abgrund auf einer neuen Ebene des Bewusstseins 
zu überwinden und die Ureinheit von Subjekt und Objekt im Zentrum der Seele wiederherzustellen.

Auf die Verwandtschaft mit Heiler und auf das Subjekt-Objekt-Denken in dieser Äusserung kommt es uns hier weniger an, als auf ihre Prämissen, die abendländische Philosophie und abendländische Gestaltungen des Christentums sind. Falls Richter etwas Wesentliches über Mystik ausgesagt haben sollte, dann muss ich allerdings gestehen, dass Bantu-Frömmigkeit diese Mystik nicht kennt.

Und weshalb denn nicht?

a) Im Wesen dieser Mystik liegt, soweit ich das überblicken kann, immer ein Selbstzweck, ihr äusserstes Ziel ist mystisches Aufgehen, und dieses ist niemals Mittel zum Zweck. Heilers „Wurzeln" dagegen, wie sie in der Bantu-Frömmigkeit vorliegen, sind meines Wissens niemals Selbstzweck, sondern immer Mittel zum Zweck. Man strebt nach der Verwischung der eigenen Bewusstseinsgrenzen, um dadurch, im Kontakt mit den Ahnen oder der Gottheit, etwas für die Gemeinschaft und ausnahmsweise für sich selbst zu gewinnen. Deshalb scheint es mir nüchtern und bezeichnend, dass z. B. M. Gelfand in seinen beiden Büchern Medicine and Magic of the Shona (1956) und Shona Religion (1962) die Mystik überhaupt nicht erwähnt.

b) Bantu-Frömmigkeit ist nicht reflektierend, abstrahierend und theoretisierend. Bantu-Philosophie ist neueren Datums und Afrikas ,,geheime Weisheit", in der das Geheimnisvolle bedeutend wichtiger ist als die Weisheit, hat mit Mystik äusserst wenig zu tun. Sie ist da um zu ängstigen und unter Kontrolle zu halten. Man denke etwa an die verschiedenen Geheimbünde und gewisse Züge in den Reifeinitiationen. Der Inhalt des Glaubens selbst wird nicht zum abstrakten Gesprächsthema, und wenn er dazu wird, so dass man in der dritten Person von ,ihnen“, d. h. den Ahnen, oder von ,ihm“, Gott, redet, geschieht das nur als Vorbereitung auf ein existentielles Gespräch mit „Ihr" und „Du“ in der 2. Person aus der Gemeinschaft heraus und in der Gemeinschaft - vielleicht wäre es richtiger zu sagen ,in der Gemeinde“. Es gibt hier keine „Objektivierung des Absoluten, das seinem Wesen nach nicht zum Objekt gemacht werden kann“"(P. Tillich, Das religiöse Symbol, nach H. Hof, Myt och symbol, I967, S. 43). Richters ,Riss“ ist auch dann nicht vorhanden, wenn zufällige Angst und Not mehr als Ehrfurcht das kommunalistische Verhältnis zu Ahnen und Gottheit bestimmen. 
c) Dieses Verhältnis ist nicht eine Angelegenheit des einzelnen, so wie wir es uns gewöhnlich in der Mystik vorstellen. Aber die Frage „Mystik und Gemeinschaft" (Rafael Gyllenberg) beschäftigt auch Europa. In der BantuFrömmigkeit ist Gemeinschaft das Grundlegende, in der Mystik das NichtIndividuelle das Peripherische.

d) Der „Riss“ existiert nicht; oder richtiger: existiert so nicht. BantuFrömmigkeit kennt kein „Mein Gott, mein Gott, warum hast du mich verlassen?" und keine Verzweiflung der Auserwählten - ebenso wenig wie den Gegenpol des mystischen Versinkens. Immerhin wäre es etwas voreilig, deshalb zu behaupten, dass die Bantu s. z. s. nicht an die Quellen gekommen sind. Man hüte sich vor abendländischen Subjektivismen!

e) Der Afrikaner ist im Gegensatz zu uns viel mehr Gemeinschaftsmensch als Individ und noch viel weniger Individualist. Gerade an diesem Punkt fällt es uns oft schwer, uns seine Bewusstseinslage recht zu vergegenwärtigen. Wir haben oben (vgl. 2a) schon die Verwischung der Bewusstseinsgrenzen erwähnt. Vielleicht kann das Wort „fluktuierend“ hier etwas vom Tatbestande vermitteln. Das Eigenbewusstsein gleitet von der einen Person in die andere und in die anderen über, und zwar nicht nur in der Gemeinschaft der jetzt Lebenden, sondern auch in der Gemeinschaft mit den Generationen der Ahnen, und aus dem positiven Lebensbewusstsein heraus noch viel weiter. Nicht „Riss", sondern stärker oder schwächer markierte Grenze, die die Zusammenschau der Aspekte nicht gefährdet. Und fängt nicht tatsächlich das Abendland wieder an, Gegensatzpaare zu überwinden und zu dieser Zusammenschau zurückzukehren? So dürfte etwa die oben erwähnte Subjekt-Objekt-Polarität in Zukunft der Sphäre gedanklicher Spekulationen entzogen werden, da sie nur von zwei verschiedenen Standpunkten zeugt. Die Zusammenschau der Aspekte lässt uns die Einheit der Welt wiedererkennen und wir sind vorsichtiger geworden im Gebrauch von solchen Gegensatzpaaren wie „Unten und Oben“, „Physik und Metaphysik“, „Geschichte und Übergeschichte“, „Zeit und Ewrigkeit" (vgl. hierzu G. Krusche, „Kirche und Kybernetik“, Zeichen der Zeit, 2/1967, 43 f.). Der Afrikaner braucht diese Umkehr nicht zu vollziehen.

f) Bewahrt hat ihn davor u. a. sein Gottesverhältnis. Er kennt nicht ,das Göttliche"; Gott ist ihm nicht transzendent und spiritualistisch. Das Verhältnis zu ihm ist „,immanent", d.h. der Gegensatz, immanent — ,trans- 
zendent" existiert nicht. Die Verbindung besteht, und zwar nicht nur dank Hypostasen und Medien, sondern auch dank Mythen und vor allem Symbolen, und das ist in der Alltagsfrömmigkeit sogar wichtiger. Die Kontinuität ist nicht ,zerrissen“. Zu beachten ist allerdings, dass trotz der sonst weit verbreiteten Besessenheit ein Mensch niemals von Mulungu, Nzambi oder Mwari, oder wie die Götter sonst noch heissen mögen, besessen wird. Die unio mystica fehlt.

\section{Mystische Züge in der Bantu-Frömmigkeit}

Wenn der Bantu-Frömmigkeit auch das Herzstück der Mystik, wie wir sie kennen, fehlt, kann man ihr trotzdem gewisse undogmatische mystische d. h. also nicht rein religiöse - Züge nicht absprechen. Sie wurzeln (ich gebrauche bewusst Richters Ausdruck) im Bewusstsein und im Erleben des Heiligen, wie es etwa die Initiationen bieten und wie es einerseits in Träumen und Visionen und andererseits in der Messiaserwartung zum Ausdruck kommt.

a) Das Heilige ... aber was ist hier mit dem „Heiligen“ gemeint? Nicht so sehr das Heilige, wie es in tempus und templum erfasst werden kann und in seiner Bantu-Entsprechung nhambo (Zeit) und -tamba (Feiern), obgleich die grosse Bedeutung der Grenze, die in diesem Paar anklingt, für die BantuFrömmigkeit und -Mystik evident ist, und es ist bezeichnend, dass -tamba im Haya „opfern“ und „,heilen“, im Vimbundu (Angola) etambo „Kultstätte" und im Karanga -tamba mhaka ,einen Rechtsfall untersuchen“ bedeutet. Das Heilige ist somit das Abgegrenzte, Abgesonderte, Besondere, Sonderbare, aber es ist nicht totaliter aliter.

Nun ist aber Mystik nicht recht denkbar ohne dieses -tamba, wo man sich aus rituellen Gründen der Arbeit enthält, wo Arbeit zum Tabu wird, das im A. T. so auffallend nicht nur die Menschen, sondern auch die Haustiere betraf. Der Feiertag ist nicht nur „Ruhetag“, sondern geradezu Unglückstag, falls an ihm gearbeitet wird, und das ist auch echt afrikanisch. Dazu kommt, dass babyl. šabattu nicht nur „Feiern“, sondern auch „Einigung“, also unio bedeuten soll (Kittel, Geschichte des Volkes Israel, 6. Aufl. 1923, I, S. 447:I).

b) Die lokale, mythologisch bestimmte Grenze im Erlebnis des Heiligen 
kommt besonders gut im Erdbodentabu zum Ausdruck. Zwei Beispiele mögen das verdeutlichen.

In einer Ursprungsmythe der Dogon (Republik Mali) hat ein Schmied des Gottes Amma im Himmel Hirse gestohlen; der erzürnte Gott lässt ihn darauf zusammen mit seiner Frau zur Erde herab. „En touchant le sol, le coupable devint impur et ne put remonter au ciel" (Tegnæus, Le héros civilisateur, 1950, 17, nach Dieterlen-de Ganay). Berührung mit der Erde führt Unreinheit im Verkehr mit Gott mit sich.

Es ist daher kein Zufall, dass das Erdbodentabu immer wieder im $\mathrm{Zu}-$ sammenhang mit der Initiation vorkommt. Die Initiandin muss auf dem Rücken getragen werden; ebenso das neue Ahnenmedium und die Braut, und auch der neugewählte Nachfolger des toten Häuptlings darf den Erdboden nicht berühren. Vom Rozwi-Herrscher in Rhodesien wurde sogar behauptet ,that if he touched the ground, heaven would fall and the world would be destroyed and calamity would befall the people". Als sein Sohn sich weigerte, das Gebot zu halten und sich deshalb Sandalen machen zu lassen, floh der Vater, von Dienern in einer Ochsenhaut getragen, zu Gott auf dessen Berg und kehrte nie wieder zurück (Sebina, „Makalaka“, Afr. Studies 6, I947, S. 83, 85-90; vgl. v. Sicard, Ngano dzeciKaranga/KarangaMärchen, I 965 , S. 235:3).

Verunreinigung hindert mystisches Erleben an der Grenze der ,rites de passage“. Aber auch dieses Tabu gilt nicht nur den Menschen, denn Dinge, die der Gottheit gehören, dürfen auch den Erdboden nicht berühren, so etwa die heilige Bundes- und Ahnentrommel Mwaris (van Warmelo, „Ngoma-Lungundu“, S. 26, in Copper Miners of Musina, Pretoria 1940; weitere Beispiele bei v. Sicard, Ngoma lungundu, I952, S. I8:2) und heilige Biergefässe; erlegtes Wild, das Mwari geopfert werden sollte, musste zu seinem Heiligtum getragen werden. Natürlich kann alles das zu kultischen Formen erstarren, auch erfahren wir nur von diesen, die Möglichkeit eines damit zusammenhängenden mystischen Erlebnisses kann jedoch nicht bestritten werden.

c) Näher rückt der Mensch in einigen Gipfelerlebnissen der Inthronisation an die mystische Grenze des Heiligen heran, weniger vielleicht noch in jenem lautlosen Stillestehen des nominierten Nachfolgers eines toten Zwimba-Herrschers an dessen Grabe, wobei er seine Hand auf einen der 
beiden Pfähle legt (es ist die symbolische Verbindung mit den Ahnen und dem Leben). Bullock (Mashona 1927, S. 284) sagt hierzu: ,,As he stands, he will feel within himself whether he is acceptable or rejected; for his beating heart will burst in his breast, if he is not in right standing at the grave."

Noch stärker kommt die mystische Grenzüberschreitung in einer Reihe von Inthronisationsriten des neugewählten Nemakonde-Häuptlings südlich vom mittleren Zambezi zum Ausdruck. Er wird an den Gräbern der Väter auf dem Berge sanktioniert. Die Szene ist von Symbolwerten geladen. „... the new Chief grasps the mbiru pole, standing in the prescribed posture with his right leg lifted, foot beneath the left knee ... he should stand in that attitude all night." Sollte sein Auftreten am Morgen zeigen, dass die Ahnen seiner Wahl zugestimmt haben, dann ist der versammelten Menge ein „unlicensed acte d'amour" gestattet, worauf der neue Häuptling in sein Gehöft getragen wird (Bullock, S. 285 f.).

Wir übergehen hier den Berg, die Nacht, den Pfahl, den acte d'amour und das Getragen-werden und heben nur die sakrale Nilotenstellung hervor. Sie ist eine Entsprechung zur kraftfördernden mythologischen Halbseitigkeit und hat es natürlich nicht ausschliesslich mit dem Jenseits oder der Totenwelt zu tun, aber doch in sehr hohem Grade. Der Nominierte steht an der Grenze, ist als Toter gewissermassen bei den Ahnen und überschreitet die Grenze aus dem Jenseits wieder zurück ins Leben. Von jetzt an aber ist er nicht mehr wie vor dieser Nacht er selbst. Kann ein Afrikaner solch eine ganze Nacht, in Symbole versunken, durchleben, ohne sich im mystischen Erleben zu verlieren?

d) Die Bedeutung des Traumes für den Afrikaner hat in letzter Zeit unter vielen andern auch B. Sundkler hervorgehoben, wobei er besonders den Gesichtspunkt der Berufung berücksichtigt (Christian Ministry I960, S. 2531). Neben dem Traum steht das Gesicht, die Vision, das Schauen. Zu den stereotypen Erscheinungen des Gesichts gehört die lichte, weisse, reine Gestalt, die die Sehnsucht nach dem Reinen erweckt, und ferner die Bergbesteigung, um Gott selbst zu begegnen. Beides darf nicht ohne weiteres aus mehr oder weniger bewusster Beeinflussung durch das Christentum erklärt werden. Denn wenn die Afrikaner das Heilige ausdrücken wollen, greifen sie wie selbstverständlich zu solchen Bildern wie ,hell, weiss, glänzend", und die Anbetung auf dem Berge ist mindestens ebenso alt, wie 
der Glaube der alten eurafrikanischen Jäger an den atmosphärischen Gott, der über Tod und Leben von Tier und Mensch waltete. Die geweckte Sehnsucht aber ist nicht ein Verlangen nach kultischer Reinheit, sondern nach einem Zustand, der ein Leben in der Nähe Gottes ermöglicht.

e) Das Besteigen des Berges kommt oft in Geschichten und Mythen vor, die dem Leben selbst entsprungen sind. Auch aus ihnen spricht Sehnsucht, zuweilen aber auch Enttäuschung. Die Erde ist nun einmal unrein, besudelt.

Eine Mythe der oben erwähnten Dogon berichtet von einem Mann, der Visionen hatte. Eines Nachts wird er von einer unbekannten Frau geweckt und auf den Gipfel des Berges geführt, wo sich ein Teich, in dem es immer Wasser gab, befand. Die Frau verschwindet im Teich und kehrt als die Gottheit Nommo mit einem Stein in der Hand zurück. Nommo gibt ihn dem Mann; es ist ein Regenstein (Dieterlen et de Ganay, Le génie des eaux chez les Dogons 1942, S. 35 f.).

In einem noch unveröffentlichten Märchen der Cokwe Angolas besucht ein wunderbar geheilter Krüppel seinen himmlischen Schwiegervater Nzambi auf dem Berge, wo dieser, umgeben von weissen Wolken, in blendendem Glanze thront. - Ein Venda-Fürst (Transvaal) besteigt den Ahnenberg und trifft dort den atmosphärischen Gott Mwari, der ihn in leuchtend weisse Gewänder kleidet (van Warmelo, S. I7), - und das soll vor kaum 200 Jahren geschehen sein! (Weitere Belege von Bergbesteigungen bei v. Sicard, Ngoma, S. 75 f.).

Es soll nicht geleugnet werden, dass auch hier in einigen Fällen atl. Beeinflussung vorliegt, aber ebenso verkehrt wäre es anzunehmen, dass die mystisch-symbolischen Erlebnisse, die diesen Erzählungen zugrundeliegen, erst mit dem A. T. zu den Bantu kamen.

Im Traum und Gesicht lag die Initiative s.z.s. bei der leuchtenden Gestalt; bei den Bergbesteigungen dagegen liegt sie oft beim Menschen selbst. In beiden Fällen aber handelt es sich um den Verkehr des Einzelnen mit der Gottheit und in beiden Fällen ist das Zusammentreffen mit Befehlen und Anordnungen für die Zukunft verknüpft (vgl. 2 a).

f) Wenn wir zuletzt einen Blick auf die alten und, soweit wir das beurteilen können, von anderen Religionen unbeeinflussten afrikanischen Messiaserwartungen werfen, berühren wir einen mystischen Zug, der uns zum afrikanischen Kommunalismus zurückführt. Der Ausdruck „Messiaserwartung“ 
ist anfechtbar, denn genau genommen kann von Messiaserwartung nur da die Rede sein, wo das A. T. wenigstens Pate gestanden hat. Die Sache selbst ist aber klar: das Volk erwartet einen Erlöser, der wiederkommen wird, um einen Idealzustand wiederherzustellen. Es weiss also nicht nur von Kulturbringern der Vergangenheit, sondern seine Hoffnung (hier weniger seine Sehnsucht) greift in die Zukunft, wobei sie sich nicht so sehr auf Prophetismus gründet, als auf den bei den Bantu tief eingewurzelten Lebensglauben, wie er etwa in der Gemeinschaft mit den Ahnen in Erscheinung tritt und in dem es ohne psychologische Konfliktsituationen nicht abgeht („Grenze!"). Vorstellungen einer vorhergehenden Katastrophe fehlen fast ganz; apokalyptische Gedanken sind eher in anderem Zusammenhang zu finden (Erdbodentabu!). Jedoch erzählen z. B. die Sonjo (zwischen dem Kilimanjaro und Victoria-See), die aus Hadramaut eingewandert sein sollen, dass bei der Rückkehr ihres Messias zwei Sonnen aufgehen würden, im Osten und im Westen, und wenn sie sich treffen, würde der Messias kommen und nach Menschen mit einem besonderen Merkmal auf der linken Brust suchen. Ihnen würde er beistehen und helfen (Fosbrooke, „Hambageu, the God of the Wasonjo“, Tanganyika Notes and Records 35/1953, 38-42).

In dem sonst so kommunalistisch orientierten Afrika wird der Messias immer als Einzelperson erwartet, jedoch nicht im abendländischen Sinne. Gerade als Messias greift er über den einzelnen hinaus. Zwischen IMensch und Mensch, und wäre der eine von ihnen auch der Messias selbst, besteht eine andere Art von Grenze, als zwischen uns und unserem Gott. Vom Bantu-Messias gilt, was Söderberg von Tammuz sagt: er sei ,,à la fois un individu et un collectif" (Religion des Cathares, I949, S. I61).

Die alte afrikanische Messiaserwartung flammte (ganz wie die moderne) oft in Krisenzeiten auf. Man stellte sich das messianische Reich gewöhnlich lokal vor. Die Erwartung konnte von dem Verlangen nach sozialer Gerechtigkeit bestimmt sein, was dann auch die Heilserwartung mitbestimmte, aber sie stand doch immer im Zeichen der Lebenserhaltung und Lebensvermehrung. Trotzdem lag die Frage nach dem ewigen Leben dieser Messiaserwartung fern, denn das Gegensatzpaar ,Zeit-Ewigkeit“ existiert nicht (vgl. 2e).

Zuweilen ist der Messias ,weiss“ (Kintu in Uganda; der letzte „Mondkönig" von Rwanda-Urundi; der weisse Messias der Konde), aber nicht wie die Lichtgestalt der Visionen, sondern wie die Albinos und Europäer. Des- 
halb wurden hier und da auch die ersten Europäer als „Messias“ begrüsst (z. B. bei den Konde). Das hängt aber höchstens sekundär mit den Weissen als ,Weisse“ zusammen, sondern entspricht eher Vorstellungen vom Ahnenund Totenreich.

Messiaserwartung und mit ihr mystische Züge sind latent im innersten Wesen Bantu-Afrikas verankert.

Abschliessend sei zuletzt noch erwähnt, dass die alte afrikanische Messiaserwartung nicht eschatologisch sondern urzeitlich ausgerichtet ist. Eine ihrer grundlegenden Voraussetzungen bildet das Bewusstsein der Notwendigkeit eines ,ZZurück zum initium“. Der Messias ist der Wiederhersteller der Lebensfülle. Der afrikanische Messias entspricht bis zu einem gewissen Grade dem paulinischen zweiten Adam (vgl. Barrett, From first Adam to last 1962, bes. S. 74). Mythologisch ausgedrückt und mystisch erlebt wurde die Gewissheit des neuen Menschengeschlechtes in den vielen, später oft zu „Verschlinge-Märchen“" herabgesunkenen Berichten vom wunderbar geborenen Drachentöter der Urzeit, der Menschen (und Tiere) aus dem Inneren des Ungeheuers befreite und einem neuen Dasein entgegenführte. 